\title{
Philosophiques
}

\section{Sur le matérialisme du jeune Marx (réponse à François Tournier)}

\section{Jean-Guy Meunier}

Volume 13, numéro 1, printemps 1986

URI : https://id.erudit.org/iderudit/203310ar

DOI : https://doi.org/10.7202/203310ar

Aller au sommaire du numéro

Éditeur(s)

Société de philosophie du Québec

ISSN

0316-2923 (imprimé)

1492-1391 (numérique)

Découvrir la revue

Citer ce document

Meunier, J.-G. (1986). Sur le matérialisme du jeune Marx (réponse à François Tournier). Philosophiques, 13(1), 164-169. https://doi.org/10.7202/203310ar d'utilisation que vous pouvez consulter en ligne.

https://apropos.erudit.org/fr/usagers/politique-dutilisation/ 
Sur le matérialisme du jeune Marx (réponse à François Tournier)

par Jean-Guy Meunier

Lorsqu'un auteur est appelé à défendre certaines de ses positions, il doit souvent inviter le critique à relire le texte et à modifier son interprétation en conséquence. Or, dans l'étude que fait François Tournier de mon livre Genèse du Matérialisme dans les écrits de Karl Marx, ce n'est pas le cas pour l'essentiel de sa lecture. Il a réussi à cerner les points importants du texte et à en expliciter les thèses principales. Plus que cela, il réussit à lancer un débat intéressant sur certains points qui n'avaient peut-être pas reçu toute l'attention qu'ils méritaient. Trois points retiendront mon attention: la nature et le statut du nominalisme, de l'explication et de la formalisation dans une théorie scientifique. 


\section{La question du réalisme et du nominalisme}

La première question que soulève Tournier touche le problème du nominalisme de Marx. Comment en effet, demande-t-il, concilier chez Marx la co-présence d'un nominalisme et d'un réalisme? Répondre à cette question implique que nous précisions le statut du langage dans une théorie et l'interprétation ontologique des expressions généralisantes.

Comme je l'ai souligné à plusieurs reprises dans mon livre, il faut se rappeler l'importance de l'aspect langagier dans la démarche de Marx. C'est une thèse répétée à satiété, mais rarement relevée par les interprètes et lecteurs de Marx, que toutes les théories qu'elles soient politiques, philosophiques, économiques, passent à travers le langage (non la langue) c.-à d. qu'elles se constituent à travers des concepts et des énoncés exprimés dans un langage.

«La réalité immédiate de la pensée est le langage» (L'Idéologie allemande, p. 59).

Aucune théorie n'est l'expression directe et isomorphique des objets dont elle parle. Les théories mettent toujours en jeu une opération langagière, qui est, comme Marx l'a montré dans l'Idéologie allemande, un lieu privilégié pour le travail idéologique. Avant même de se demander si une théorie est vraie ou fausse, il faut voir comment elle fonctionne dans sa structure langagière.

Une fois cependant qu'on accepte l'importance du langage dans la théorie, reste à savoir quel statut théorique ont les expressions de ce langage. Si un langage théorique met en jeu des expressions ou des termes et par conséquent s'ils expriment des concepts et des idées, comment doivent-ils être interprétés sur le plan ontologique? Ne référent-ils quà des choses, des entités ou substances empiriques individuelles (positions nominalistes) ou réfèrent-ils à des substances générales (positions réalistes) ? La question est importante pour la théorie marxiste parce que, d'une part, si toutes les expressions d'un langage théorique réfèrent à des réalités ou des substances, alors les expressions générales aussi le feront. Si des noms comme "Bauer», "Feuerbach » réfèrent concrètement à des individus réels concrets, les expressions comme «idéologie», «économie», «argent», «capital» ont-elles un statut analogue? Ces termes généraux nomment-ils des substances?

À l'inverse, si ces expressions générales ne sont pas interprétables en termes réalistes, ne sont-elles que des abstractions sans lien avec la réalité? Les concepts théoriques que Marx met en place comme «classes sociales», «prolétariat», «bourgeoisie», etc. ne sont-ils que des «abstractions» ?

Poser la question en ces termes généraux, c'est placer Marx au cœur du débat nominalisme/réalisme. Il faut cependant, pour voir plus clair dans ce débat, le reformuler avec un plus de précision et lui donner au moins la formulation qu'il a dans le débat technique. Dans ce débat, la question 
centrale porte sur le statut ontologique des expressions du langage et plus spécifiquement sur l'interprétation à donner aux expressions nominales et aux expressions prédicatives. Or, le nominalisme et le réalisme ont des réponses assez similaires sur la fonction sémantique des expressions référentielles du type nom-propre ou variable. Ces expressions nomment des individus dans l'univers de référence.

Le véritable lieu cependant de l'opposition entre le réalisme et le nominalisme est la question des prédicats, c.-à $\mathrm{d}$. des expressions généralisantes, celles où précisément s'inscrivent les «idées» ou les « concepts». Il y a là ferme opposition. Chacun n'accorde pas un même statut ontologique au référent de ces expressions. Pour les réalistes, ces expressions sont interprétables en termes d'entités et de substances appartenant à un univers d'ordre abstrait ou logique. Pour les nominalistes, les prédicats sont interprétables en termes d'opérations sur les individus. Ils ne désignent aucun objet ou substance particulière de l'univers concret ou abstrait.

Or Marx prend une position ferme dans ce débat. Il prend la position réaliste et (évidemment nominaliste) quant à l'interprétation des expressions du type nom-propre ou variable. Car il est clair, pour Marx, qu'il y a « du réel » au-delà de la conscience et donc qu'il existe des objets et des individus empiriques. Mais sa position nominaliste porte sur le statut des expressions du type prédicat ou «concept» c'est-à-dire les expressions généralisantes. Pour lui, ces types d'expressions nomment non pas des substances mais des rapports et des relations. Les référents de ces expressions ne sont jamais des substances autonomes. Leur fonction dans la théorie est de nommer des rapports :

«Un rapport, ce que les philosophes appellent une idée» (Idéologie Allemande, p. 107)

«Si ces concepts généraux prennent valeur de puissances mystérieuses, c'est la conséquence nécessaire du fait que les rapports dont ils sont l'expression sont devenus autonomes» (Ibid, p. 399)

«Dans le langage, tout rapport ne peut s'exprimer que sous forme de concept» (Ibid, p. 399)

Ainsi, le conflit entre réalisme et nominalisme chez Marx n'est-il qu'apparent. Il est réaliste sur le plan des expressions d'individus, position qui n'est pas contradictoire avec celle des nominalistes. Il est cependant clairement nominaliste quant au statut des expressions prédicatives et généralisantes. Cette position est très bien illustrée par l'analyse que Marx fait du concept d'bomme et d'bumain. Il est évident que pour Marx ce qui est existant ce sont les individus-hommes. Mais le concept d'homme et d'bumain réfère non a des substances, mais à des rapports entre ces individus : miné...»

— «l'expression positive "humain" correspond à un système déter- 
- «Cette somme de forces productives, de capitaux, de formes, de relations sociales, que chaque individu et chaque génération trouve comme données existantes, est la base concrète de ce que les philosophes se sont représenté comme substance et "essence" de "l'homme"》(Manuscrits de 1844 , p. 89 ).

— «Donc d'emblée se manifeste un système de liens matériels entre les hommes... système de liens qui prend sans cesse de nouvelles formes...» (Ibid, p. 59)

Ces rapports n'«existent» pas en dehors et au-delà de l'homme concret. Ils n'ont pas d'existence autonome. Ils ne sont que nommés dans les théories. Ainsi, pour Marx, toutes les expressions générales ou les concepts ne nomment pas des substances, mais des rapports entre individus concrets. Il ne peut être que nominaliste. Je ne vois pas d'ailleurs comment la structure conceptuelle et l'argumentation de Marx pourraient être différentes. Comment Marx pourrait-il, de manière cohérente, donner un statut ontologique de type substance à ces concepts généraux sans tomber dans une métaphysique. Et surtout je ne vois pas comment il pourrait faire jouer à ces expressions les rôles sémantiques et logiques qu'il leur fait jouer dans l'ensemble de sa théorie. [Cf., toutes les relations d'identité qu'il établit entre les concepts au sein de la théorie économique.] Ceci ne serait pas possible si ces concepts étaient interprétés de manière réaliste.

Bref, nous pourrions dire que Marx maintient un discours réaliste (acceptable par ailleurs pour les nominalistes) quant au statut des expressions d'individus, mais une position nominaliste quant au statut des expressions généralisantes. Mais pour démontrer davantage cette thèse, il faudrait une étude plus approfondie des textes.

\section{Sur la pratique scientifique de Marx}

Le deuxième point important que souligne Tournier touche la question du type de pratique scientifique que je prête à Marx. Selon Tournier, les positions que je tiens sur la critique de l'empirisme naïf et du formalisme de la méthode de Marx sont discutables.

En effet, Tournier croit que j'ai tort de placer Russell et Popper dans la classe-type des empiristes naïfs que critique Marx: «(Ceux-ci) ne sont pas aussi naïfs qu'on voudrait le faire croire» (Tournier). Pour répondre à cette critique, il faut préciser dans quel contexte Marx parle de l'empirisme naif. Ce que Marx reproche aux « positivistes» de son époque et ce qui pourrait être reproché aussi à certains praticiens contemporains de la science est leur tendance à prendre l'observation contrôlée et surtout le discours descriptif dans lequel elle s'explicite pour une explication du phénomène. C'est à cet empirisme naïf que Marx s'oppose.

Or, j'ai comparé cet empirisme naïf que critique Marx avec certaines positions épistémologiques contemporaines, mais cela ne survient que sur la 
question de l'observation et de la description et non de l'explication dans le discours scientifique. Et il n'est pas question de situer Popper et Russell dans cet empirisme naïf. Si j'ai repris ces auteurs, c'est pour montrer que sur cette question spécifique de l'explication, c'est-à-dire là où précisément l'empirisme naïf est dépassé, il y a une différence théorique importante entre Marx et ces épistémologues contemporains. Cette question dans cette épistémologie s'explicite dans les termes de la vérification (Ayer) et de la falsification (Popper) alors que chez Marx elle ne semble pas (du moins dans les écrits de jeunesse) s'orienter dans une telle voie. En effet, bien que les textes de cette époque soient relativement silencieux sur cette question, on peut voir, par plusieurs remarques critiques de Marx, que sa théorie de «l'explication» se déploie beaucoup plus vers la nature et le contenu des propositions que sur la question de leur validation. Pour Marx, un discours explicatif est celui qui se caractérise avant tout par l'analyse des rapports et des relations au sein de la réalité.

«Pour Marx, ce que la science doit démontrer ce sont des "liens", des "rapports", des "relations". Un discours scientifique est la mise en rapport de concepts et de propositions qui doivent exprimer des relations matérielles (...) C'est la structure des relations dans un phénomène qui est l'essentiel et non ce que l'expérience immédiate "positive" pourrait en saisir». (Meunier, p. 112).

En d'autres termes, pour Marx, le débat épistémologique sur l'explication ne porte pas sur une opposition à des critères de validation (ce n'est pas une question qu'il pose) mais sur la nature des propositions scientifiques c.-à d. leur contenu. C'est une focalisation différente de la problématique.

\section{À propos de la méthode analytico-constructiviste}

Abordons maintenant un dernier point important relevé par Tournier. Il semble que la position « analytico-constructiviste» que j’ai prêtée à Marx le situe dans une démarche formalisante qui ne lui sied aucunement et que cela serait même une lecture très idéologique de Marx. On tomberait ainsi dans le reproche d'hypersymbolisation si propre à la normativité épistémologique contemporaine.

"L'épistémologie "analytico-constructiviste" de Meunier est tout à fait dans le ton de cette "idéologie" de la science dont Popper voit croître la popularité non sans entretenir certaines craintes.» (Tournier)

Cette remarque est intéressante, car elle me permet de préciser la question du formalisme dont il est question dans le dernier chapitre. Il y a une nette différence entre formuler un problème en termes de rapports et de relations et formaliser dans un symbolisme ces rapports et ces relations. Et sur cette question Marx est clair. Il insiste en effet sur la nécessité pour une théorie de décrire les relations et les rapports, mais il ne dit jamais qu'il est nécessaire de les exprimer dans un formalisme ou une symbolisation. Au 
contraire Marx craint le formalisme et le jeu des formules qui lui sont propres (cf. sa critique de Bauer dans L'Idéologie allemande). Il y a là une différence nette entre le discours marxiste et certains discours scientifiques contemporains. Tant pour Marx que pour la science contemporaine, l'analyse des rapports et des relations est une priorité. "Autant pour Marx que pour l'épistémologie contemporaine, la science n'est pas simplement identification et classification des phénomènes.» (Meunier, p. 178) Mais la différence entre les deux est dans le type de langage utilisé pour parler de ces rapports et relations. Pour Marx, il n'est aucunement question de privilégier le formalisme ou la symbolisation. Tout au plus y a-t-il formulation mais non formalisation. Alors que dans la science contemporaine le formalisme est synonyme de formulation adéquate.

Il m'apparaît évident que le formalisme entendu comme symbolisation univoque et non ambiguë n'est pas le seul langage ou discours qui puisse décrire les rapports et les relations de l'univers matériel. Théoriquement, on peut penser qu'un bon formalisme peut exprimer adéquatement des rapports et des relations (cf. les prédicats relationnels, les opérateurs, etc.). Mais certains de ces rapports sont si complexes qu'il devient difficile de les exprimer dans des formules simples et univoques. Le langage naturel, à cause de sa capacité de généralisation et d'abstraction, est souvent plus en mesure d'exprimer adéquatement la structure de ces rapports. Certes, il peut être ambigu et prêter le flanc à des interprétations diverses. Mais il possède aussi des moyens de correction et de contrôle pour arriver à ces fins. Le formalisme n'est pas le fin mot de toute science. Même les écrits mathématiques les plus formels sont introduits en langage naturel!

Bref, la méthode « analytico-constructiviste» que nous prêtons à Marx ne signifie aucunement un privilège accordé au formalisme et à la symbolisation. Cette méthode signifie essentiellement qu'une démarche théorique de type scientifique, dans un premier temps, doit (via l'analyse) se soucier du langage dans lequel la réalité est exprimée et deuxièment elle doit centrer son attention sur les rapports et relations existant dans cette réalité et ceci dans une finalité pragmatique de transformation de cette réalité.

Département de philosophie

Université du Québec à Montréal 